\title{
Anti Doping Administration \& Management System (ADAMS)
}

\section{Dopingkontrollsystem}

In Deutschland erfolgt die Planung, Koordination und Weiterentwicklung des Dopingkontrollverfahrens im Bereich des Spitzensports durch das Ressort Doping-Kontroll-System (DKS) der NADA. ${ }^{1}$ Im Rahmen dessen wird die Organisation der Kontrollen sowohl außerhalb aber auch innerhalb des Wettkampfes (Trainingsund Wettkampfkontrollen) organisiert. ${ }^{2}$ Sofern ein Athlet zu denen gehört, bei denen Trainingskontrollen durchgeführt werden, können diese unangekündigt zu jeder Zeit außerhalb eines Wettkampfes erfolgen. Dabei sind Kontrollen an jedem dazu geeigneten Ort denkbar, so dass weder die eigene Wohnung, noch die Trainingsstätte oder die Schule davon ausgeschlossen sind. Wer einem Testpool der NADA angehört, muss demnach jederzeit mit einer Dopingkontrolle rechnen und dafür entsprechend zur Verfügung stehen. ${ }^{3}$

Damit die Sportler von den Kontrolleuren aufgefunden werden können, müssen die Athleten aus dem ATP nach Erhalt der Testpoolbenachrichtigung auf einem auf der Homepage der NADA abrufbaren Athleten-Meldeformular ${ }^{4}$ den Wohnsitz und sonstige Aufenthaltsorte, wie Schule, Universität usw., die E-Mail-Adresse, Festnetz- und Mobilfunknummer sowie Ort und Zeit des Trainings in Form eines Rahmentrainingsplans angeben. ${ }^{5}$ Den Sportler trifft danach nicht nur die Verpflichtung seine Stammdaten sowie seine Adresse anzugeben, sondern er muss außerdem einen Wochenplan (Rahmentrainingsplan und regelmäßigen Tätigkeiten des Athleten), Angaben zur Gültigkeitsdauer und Saisonhöhepunkte machen. Zentrales Element der Erhebung und Auswertung von Aufenthalts- und Erreichbarkeitsinformationen der Sportler außerhalb eines Wettkampfes stellt das Anti Doping Administration \& Management System (sog. ADAMS) dar. ${ }^{6}$

Entsprechend dem Artikel 5.5.2 des NADA-Code 2015 sollen Dopingkontrollen soweit möglich über ADAMS oder einem anderen Datenverarbeitungssystem koordiniert werden, das dann aber vom WADA anerkannt sein muss. ${ }^{7}$

Beim ADAMS handelt es sich laut der dem NADA-Code von 2015 angefügten Begriffsbestimmung (Anhang 1) um ein webbasiertes Datenmanagementsystem für Dateneingabe, Datenspeicherung, Datenaustausch und Berichterstattung, mithilfe dessen eine Unterstützung der WADA und sonstiger Berechtigter bei deren Anti-Doping-Maßnahmen unter Einhaltung des Datenschutzrechts bezweckt wird. ${ }^{8}$

1 Vgl. Beschreibung unter http://www.nada.de/de/doping-kontroll-system/\#. VwtwSHrzDb4 (Abruf aller Seiten am 28.10.2017).

2 Vgl. unter http://www.nada.de/de/doping-kontroll-system/\#.VwtwSHrzDb4.

3 Zum Ganzen siehe NADA (Hrsg.), Athletenbroschüre, 2016, S. 15.

4 http://www.nada.de/fileadmin/user_upload/nada/DKS/140128_AthletenMeldeformular.pdf.

5 Dazu auch die NADA (Hrsg.), Athletenbroschüre, 2016, S. 17.

6 Zugriff über https://adams.wada-ama.org/adams/.

7 Vgl. Art. 5.5.2. NADA-Code 2015, abrufbar unter http://www.nada.de/ fileadmin/user_upload/nada/Downloads/Regelwerke/NADA-Code_2015.pdf.

8 Anhang 1 des NADC 2015, Begriffsbestimmungen; abrufbar unter http:// www.nada.de/fileadmin/user_upload/nada/Downloads/Regelwerke/NADA-Co-
Mithilfe des Systems soll den Sportlern in jedem Land jederzeit die entsprechende Nutzung elektronischer - ggf. mobiler Endgeräte zum Zweck der Aktualisierung seiner Aufenthaltsinformationen ermöglicht werden. ${ }^{9}$ Hintergrund ist, dass nach Auffassung der beteiligten Stellen die effektive Planung und Organisation eines Dopingkontrollverfahrens erst durch das Wissen über den konkreten Aufenthaltsort eines Sportlers zu einem bestimmten Zeitpunkt ermöglicht wird. ${ }^{10}$

\section{Datenschutz}

Das internetbasierte ADAMS wird durch die WADA selbst als sogenanntes "Clearinghouse“ bzw. in der Übersetzung als „Clearingstelle" von Kanada (Montreal) aus betrieben. ${ }^{11}$ Inzwischen gibt es zahlreiche Forderungen bzw. Bestrebungen, zum Teil durch die Sportler selbst, die Funktion von ADAMS zu erweitern und die Aufenthaltsorte sogar jederzeit mithilfe elektronischer Erfassungssysteme (bspw. mithilfe von GPS - Global Positioning System) zu bestimmen. ${ }^{12}$ Neben den gesammelten Stammdaten, stehen dadurch dann außerdem jederzeit die Standortdaten des Athleten zur Verfügung. ${ }^{13}$ Neben dieser Vielzahl an zu erhebenden Daten zur Dopingkontrolle ist zudem zu bedenken, dass zur Leistungsbeurteilung und zur Wettkampf- bzw. Trainingsanalyse inzwischen der Sportler ebenfalls eine Vielzahl an Informationen zur Verfügung und sich dadurch einer rundum Überwachung ausgesetzt sieht. ${ }^{14}$ Die damit verbundene Möglichkeit, zur Profilbildung auf eine Fülle von Daten zugreifen zu können, ist demnach überhaupt nicht ausreichend gesetzlich geregelt. ${ }^{15}$

de_2015.pdf; zur Kritik der Sportler an dem zu komplizierten und zu wenig benutzerfreundlichen Umgang mit ADAMS vgl. bei Musiol, SpuRt 2009, S. 90 (91).

9 Dazu unter https://www.nada.at/de/kontrolle/dopingkontrolle/adams; zu Ansätzen diese außerdem mit Aufenthaltsermittlungsgeräten zu ergänzen vgl. den Ansatz in der Leichtathletik, Bericht von Morrissey, Lokalisierung mit „eves": Mehr Sicherheit im ADAMS-System, vom 26.9.2014, abrufbar unter https://www.leichtathletik.de/news/news/detail/lokalisierung-mit-eves-mehr-sicherheit-im-adams-system/

10 Sogar von der entscheidenden Grundlage des Dopingkontrollverfahrens ausgehend, Mortsiefer, Datenschutz im Anti-Doping-Kampf, S. 51.

11 So als Begriff von der WADA selbst genutzt, vgl. WADA-Code von 2015, Art. 14.5. und 18.4., abrufbar unter https://www.wada-ama.org/en/resources/the-code/world-anti-doping-code; zur Übersetzung siehe die inoffizielle Fassung unter https://www.nada.at/files/doc/Regelwerke/2015-wadc-final-de.pdf.

12 Deutsch auch als Globales Positonsbestimmungssystem bezeichnet, vgl. zum Vorteil den Sportler sich erhoffen, zum einen den Beitrag von Morrissey, Lokalisierung mit "eves": Mehr Sicherheit im ADAMS-System, vom 26.9.2014, abrufbar unter https://www.leichtathletik.de/news/news/detail/lokalisierungmit-eves-mehr-sicherheit-im-adams-system/; zum anderen vgl. auch die Kritik in dem Bericht "Spitzensportler kritisieren Doping Meldesystem Adams" der Thüringer Allgemeinen vom 25.12.2011 und der Bereitschaft der Sportler GPS-Fußfesseln zu akzeptieren, abrufbar unter http://www.thueringer-allgemeine.de/ web/zgt/sport/detail/-/specific/Spitzensportler-kritisieren-Doping-Meldesystem-Adams-1375608553; siehe aber zur Kritik und Möglichkeiten anderer Kontrollmechanismen vor allem auch die Beiträge in diesem DuD, Heft 12/2017, S. 735.

13 Zur Kritik und Möglichkeit anderer Lösungen siehe Herber, DuD 2017, Heft 12 , in diesem Heft.

14 Vgl. dazu ausführlich bei Börding/v. Schönfeld, SpuRt 2016, S. 7.

15 Dazu schon Röhl, SpuRt 2012, S. 137. 